\section{Fatores associados à dor musculoesquelética em escolares da rede pública municipal no extremo sul do Brasil}

\section{Factors associated with musculo-skeletal pain in municipal public school students in the far South of Brazil}

\begin{abstract}
Objectives: to identify the factors associated with musculo-skeletal pain (MSP) in schoolchildren in municipal public schools in the city of Rio Grande, in the far south of Brazil, between June and December 2010.

Methods: a sectional study was carried out with a random sample of 625 schoolchildren aged between six and eighteen years of age. MSP was investigated using the Nordic Osteomuscular Symptoms Questionnaire (NOSQ) and information relating to demographic and biological factors, the means of transport of school materials and lifestyle, obtained using a standardized questionnaire. The raw and adjusted prevalence rations for association between MSP and the factors under study were estimated using a Poisson regression model.

Results: the prevalence of MSP was $37.6 \%$. Being aged between 11 and 18 years $(P R=1.70$; $C 195 \%=1.36-2.11 ; p<0.001)$, being female $(P R=1.47$; $C 195 \%=1.19-1.81 ; p<0.001)$ and a family history of back pain $(P R=0.78 ; C 195 \%=0.64-0.96 ; p=0.020)$ were associated with the outcome. Not practicing competitive sports was a protective factor $(P R=0.65$; CI95\%=0.51-0.81; $p<0.001)$.

Conclusions: these results confirm the negative influence of various factors present in the school environment on the health of students. It is considered an important measure to introduce health promotion programs in schools to encourage students to adopt healthy practices that minimize the deleterious effects of MSP.
\end{abstract}

Key words School health, Health promotion, Musculoskeletal pain
Mirelle de Oliveira Saes 1

Marcela Dupont Soares 2

Ana Mucillo-Baisch 3

Maria Cristina Flores Soares 4

1-4 Universidade Federal do Rio Grande. Rua Visconde de Paranaguá, 102 Centro. Campus Saúde. Rio Grande, RS, Brasil. CEP: 96.203-900. E-mail: mirelle_saes@yahoo.com.br

\section{Resumo}

Objetivos: identificar os fatores associados à dor musculoesquelética (DME) em escolares da rede municipal de ensino na cidade de Rio Grande, extremo sul do Brasil, entre junho e dezembro de 2010.

Métodos: trata-se de um estudo seccional conduzido em uma amostra aleatória de 625 escolares entre seis e 18 anos de idade. A DME foi pesquisada utilizando o Questionário Nórdico de Sintomas Osteomusculares (QNSO) e informações demográficas, biológicas, forma de transporte do material escolar e de estilo de vida obtidas via questionário padronizado. As razões de prevalência bruta e ajustada da associação da DME com os fatores investigados foi estimada em um modelo de regressão de Poisson.

Resultados: a prevalência de DME foi de 37,6\%. Idade entre 11 e 18 anos $(R P=1,70 ;$ IC95\%=1,36$2,11 ; \quad p<0,001), \quad$ sexo feminino $(R P=1,47$; IC95\%=1,19-1,81; $p<0,001)$ e histórico familiar de distúrbio na coluna $(R P=0,78$; IC95\%=0,64-0,96; $p=0,020$ ) estiveram associados ao desfecho. Não praticar esportes de competição foi um fator de proteção $(R P=0,65 ; I C 95 \%=0,51-0,81 ; p<0,001)$.

Conclusão: estes resultados confirmam a influência negativa de diferentes fatores presentes no ambiente escolar sobre a saúde dos estudantes. Considera-se como medida importante a implantação de programas de promoção da saúde nas escolas, que estimulem entre os estudantes a adoção de práticas saudáveis que minimizem os efeitos deletérios da $D M E$.

Palavras-chave Saúde escolar, Promoção da saúde, Dor musculoesquelética 


\section{Introdução}

A dor musculoesquelética (DME) pode ser conceituada como qualquer alteração que gere uma situação de dor em determinada parte do corpo. ${ }^{1}$ Antigamente, somente era dada importância para a presença da DME associada a uma doença subjacente, sem considerar a origem multifatorial da dor e sua presença sem associação com alguma patologia específica. ${ }^{2}$

A prevalência de DME entre escolares varia entre $12,2-30,4 \%$ em estudos nacionais e internacionais. ${ }^{3-6}$ A literatura relata que a prevalência de DME nesta população é elevada e vem agravandose, podendo causar impacto sobre a prestação de cuidados à saúde, funcionalidade e participação do indivíduo na sociedade. 7,8

Embora a DME possa ser atribuída a diferentes causas, caracterizando-a como uma condição multifatorial, 8 pesquisas têm mostrado que muitas lesões musculoesqueléticas têm fatores de risco comuns, tais como idade, ${ }^{4}$ sexo, 6 raça, 9 excesso de peso da mochila escolar, 3 sedentarismo, 5 elevado índice de massa corporal10,11 e tabagismo.

Evidências demonstram que a presença de DME em duas ou mais regiões corporais é frequente entre escolares, sendo mais referidas as regiões da coluna vertebral, ${ }^{6}$ membros inferiores ${ }^{13}$ e pescoço. ${ }^{14}$ Indivíduos com DME buscam mais atendimento por profissional da área da saúde e utilizam mais medicamentos para dor, o que acarreta uma modificação das atividades rotineiras, afetando assim sua qualidade de vida. 15

Investigar a prevalência da dor musculoesquelética em escolares e identificar os fatores que contribuem para o seu aparecimento oferece subsídios para o fortalecimento e desenvolvimento das politicas públicas na escola, voltadas para ações que considerem a influência dos diversos fatores relacionados ao ambiente em que os estudantes estão inseridos, e não somente suas características individuais. ${ }^{16}$

Sendo assim, este estudo se justifica pela importância de conhecer a situação vivenciada pelos escolares, identificando os elementos associados ao ambiente escolar que influenciam a sua condição musculoesquelética. Desta forma, o presente estudo teve o intuito de avaliar a prevalência de DME e seus fatores determinantes em escolares da rede pública de ensino de um município do extremo sul do Brasil.

\section{Métodos}

Trata-se de um estudo epidemiológico transversal analítico, que teve como público-alvo escolares matriculados entre o $1^{\circ}$ e o $9^{\circ}$ ano de quatro escolas municipais da cidade de Rio Grande/RS. O município de Rio Grande está situado no extremo sul do Brasil, com uma população aproximada de 206.161 pessoas, desta, 57.563 são usuários da rede de ensino, distribuídos em 96 escolas de ensino fundamental (15 privadas, 28 estaduais e 53 municipais); 21 de ensino médio (sete privadas, 13 estaduais e um federal) e 77 pré-escolas (18 privadas, 17 estaduais e 42 municipais). ${ }^{17}$

Foram incluídos no estudo escolares com idade entre seis e 18 anos, com condições cognitivas de compreender e responder ao instrumento de coleta de dados e que não apresentassem diagnóstico médico de patologia musculoesquelética.

O tamanho da amostra foi calculado utilizando como parâmetros uma prevalência de DME de 30\%, erro alfa de $5 \%$ e uma margem de erro de $4 \%$, o que totalizou 504 escolares. Ao acrescentarmos 10\% para perdas, o total da amostra foi 554 escolares. Para o estudo de associação considerou-se um poder estatístico de $80 \%$, um erro alfa de $5 \%$, um risco relativo maior ou igual a 1,45 e uma prevalência entre os não-expostos (variável excesso de peso na mochila) de $28 \%$, totalizando 474 escolares. Assim, acrescentando-se $10 \%$ para perdas e $15 \%$ para controle de fatores de confusão foi identificada a necessidade de uma amostra total de 599 escolares.

O processo amostral foi realizado em dois estágios. No primeiro, foram selecionadas as escolas a partir de uma lista de instituições de ensino fundamental que tinham vínculo com projetos da Universidade Federal do Rio Grande e que estivessem distribuídas em diferentes bairros do município. Posteriormente, os escolares foram sorteados a partir da lista de chamada de cada turma, guardando-se a proporcionalidade em relação ao número total de crianças matriculadas e ao número de turmas existentes em cada escola.

A coleta de dados foi realizada na escola, entre junho e dezembro de 2010, no turno de atividade escolar, em local reservado, previamente definido pela direção da escola. Os dados foram coletados por dois avaliadores devidamente treinados, sendo um responsável pela primeira etapa da coleta, que consistiu na aferição de peso e altura corporal e peso da mochila, utilizando balança antropométrica mecânica de $150 \mathrm{Kg}$, sem calçados e com o mínimo de vestimentas, e o segundo, responsável pela aplicação dos instrumentos de coleta de dados.

Informações sobre DME foram obtidas por meio do Questionário Nórdico de Sintomas Osteomusculares (QNSO) simplificado e adap- 
tado, 18 excluindo-se as variáveis relativas à situação ocupacional, e com o auxílio de uma ilustração do corpo humano. Informações sobre outros fatores potencialmente associados ao DME, não incluídos no QNSO, foram obtidas por meio de outro questionário padronizado.

O QNSO é um instrumento quantitativo que identifica a presença, localização e magnitude dos sintomas osteomusculares. 18 Originalmente, o instrumento avalia a presença de DME nos 12 meses e sete dias anteriores à entrevista. Contudo, buscando-se evitar um possível viés de memória, considerou-se neste estudo a presença de sintomas nos sete dias que antecederam a entrevista.

Definiu-se como presença de DME, a queixa de DME, referida pelo escolar, em pelo menos uma das seguintes regiões do corpo: pescoço/região cervical, ombros, punho/mãos/dedos, coluna dorsal, coluna lombar e quadril/membros inferiores.

$\mathrm{O}$ estado nutricional foi classificado de acordo com o índice de massa corporal IMC/idade, 19 sendo categorizado em: baixo peso (percentil $<5$ ), eutrófico (percentil $\geq 5$ e $\leq 85$ ); sobrepeso/obeso (percentil $\geq 86$ ). Esta escala de estado nutricional leva em consideração o peso, altura, idade e sexo da criança/adolescente.

$\mathrm{O}$ instrumento de transporte do material escolar pelo estudante foi classificado segundo o tipo (mochila com alças, carrinho e pasta de mãos/ um ombro só); modo de transporte (costas - 2 alças, costas - 1 alça e braços/mãos) e o peso do material (razão entre o peso do material escolar e o peso do estudante), que foi categorizado em adequado (razão $\leq 10 \%$ ) ou inadequado (razão $>10 \%$ ).

As variáveis de exposição foram organizadas seguindo modelo hierárquico e, em cada nível, foram ajustadas entre si e para as variáveis de níveis superiores, por meio de seleção para trás, considerando um valor de $p<0,20$ para inclusão das variáveis durante a análise ajustada, nível a nível. O modelo de análise foi composto como segue: $1^{\circ}$ nível Características demográficas (idade, sexo, cor); $2^{\circ}$ nível - Determinantes biológicos (histórico familiar de dores na coluna, situação nutricional); $3^{\circ}$ nível Fatores associados à escola (tipo de suporte e modo de transporte do material escolar, peso da mochila escolar); estilos de vida (práticas sedentárias, realização de atividade física na escola, prática de atividade física fora do ambiente escolar, participação em esporte, esporte de competição, tabagismo).

Utilizaram-se o programa Epi-Info 6.04 e STATA 10.0 para a entrada, consistência e análise dos dados. Inicialmente foi verificada a distribuição de frequência das variáveis. A magnitude da associação entre DME e as variáveis de independentes foi estimada pelo cálculo da razão de prevalência bruta e ajustada e seus respectivos intervalos de confiança (95\%) pelo método da regressão de Poisson com estimativas de variância robusta. As variáveis estatisticamente associadas ao desfecho com valor de $p<0,05$ foram mantidas no modelo final.

$\mathrm{O}$ estudo atendeu às diretrizes da resolução 196/96 do CNS tendo sido aprovado pelo Comitê de Ética em Pesquisa na Área da Saúde da Universidade Federal do Rio Grande (CEPAS-FURG), sob número 20/2010

\section{Resultados}

A Tabela 1 descreve as características dos escolares e a distribuição das variáveis independentes conforme o desfecho estudado. Dos 650 escolares elegíveis para o estudo $2,5 \%(\mathrm{n}=16)$ recusaram-se a participar e $1,5 \%(n=9)$ foram classificados como perda, chegando a uma amostra final de 625 escolares. Cerca de $50 \%$ eram do sexo feminino e tinham idade entre 11 e18 anos. Aproximadamente 70\% eram de cor branca e em torno de $40 \%$ apresentavam história familiar de dor musculoesquelética.

Com relação à condição nutricional dos escolares avaliados, maior parte era eutrófica e quanto ao tipo de suporte para o transporte do material escolar, a maioria usava mochila com duas alças carregada nas costas.

Dos escolares avaliados, $29,7 \%$ transportavam material escolar com peso superior a $10 \%$ do seu peso corporal.

Em relação aos hábitos de vida, verificou-se que $22,9 \%$ realizavam pelo menos uma das práticas sedentárias investigadas. Destes, $64,6 \%$ referiram permanecer três horas ou mais por dia assistindo televisão; 31,2\% relataram este mesmo tempo em frente ao computador e $23,3 \%$ jogando videogame.

Cerca de $80 \%$ dos entrevistados praticavam educação física na escola. A frequência desta prática era diferente entre os escolares, podendo ser três $(7,8 \%)$, duas $(46,5 \%)$, ou uma $(45,7 \%)$ vez por semana.

Entre os 625 escolares examinados, 235 relataram DME em pelo menos uma das regiões investigadas, obtendo-se uma prevalência de 37,6\% (IC95\% 33,7 - 41,4). Entre os que referiram DME, os locais mais prevalentes foram: punhos/ mãos/dedos $(12,2 \%$; $n=29)$, quadril/membros inferiores $(12,2 \% ; n=29)$, ombros $(11,2 \% ; n=26)$, região dorsal da coluna vertebral $(11,0 \% ; n=26)$, região lombar $(10,9 \% ; n=26)$ e pescoço/região cervical $(10,1 \% ; n=24)$. 
Tabela 1

Prevalência da dor musculoesquelética (DME) segundo características sociodemográficas, antropométricas e clínicas em escolares do $1^{\circ}$ ao $9^{\circ}$ ano do município de Rio Grande, RS, 2011.

\begin{tabular}{|c|c|c|c|c|c|c|c|}
\hline \multirow[t]{3}{*}{ Variável } & \multirow[t]{3}{*}{ N } & \multirow[t]{3}{*}{$\%$} & \multicolumn{4}{|c|}{ DME } & \multirow[t]{3}{*}{$p$} \\
\hline & & & \multicolumn{2}{|c|}{ Sim } & \multicolumn{2}{|c|}{ Não } & \\
\hline & & & $\mathrm{n}$ & $\%$ & $\mathrm{n}$ & $\%$ & \\
\hline Idade (anos) & & & & & & & $<0,001$ \\
\hline $6-10$ & 278 & 44,5 & 76 & 27,3 & 202 & 72,7 & \\
\hline $11-18$ & 347 & 55,5 & 159 & 45,8 & 188 & 54,2 & \\
\hline Sexo & & & & & & & 0,001 \\
\hline Feminino & 341 & 54,6 & 149 & 43,7 & 192 & 56,3 & \\
\hline Masculino & 284 & 45,4 & 86 & 30,3 & 198 & 69,7 & \\
\hline Cor da pele & & & & & & & 0,586 \\
\hline Branco & 452 & 72,3 & 167 & 36,9 & 285 & 63,1 & \\
\hline Não-branco & 173 & 27,7 & 68 & 39,3 & 105 & 60,7 & \\
\hline Histórico familiar dores & & & & & & & 0,001 \\
\hline \multicolumn{8}{|l|}{ na coluna } \\
\hline Sim & 265 & 42,7 & 119 & 44,9 & 146 & 55,1 & \\
\hline Não & 356 & 57,3 & 113 & 31,7 & 243 & 68,3 & \\
\hline Condição nutricional & & & & & & & 0,265 \\
\hline Eutrófico & 398 & 63,8 & 160 & 68,1 & 238 & 61,2 & \\
\hline Baixo peso & 21 & 3,4 & 6 & 2,9 & 15 & 3,9 & \\
\hline Sobrepeso & 110 & 17,6 & 40 & 17,0 & 70 & 18,0 & \\
\hline Obesidade & 95 & 15,2 & 29 & 12,3 & 66 & 17,0 & \\
\hline Tipo de suporte do & & & & & & & 0,018 \\
\hline \multicolumn{8}{|l|}{ material } \\
\hline Com alças & 504 & 80,9 & 183 & 36,3 & 321 & 63,7 & \\
\hline Carrinho & 45 & 7,2 & 12 & 26,7 & 33 & 73,3 & \\
\hline Pasta de mãos/Um Ombro & 74 & 11,9 & 40 & 54,1 & 34 & 45,9 & \\
\hline Modo de transporte & & & & & & & 0,153 \\
\hline Costas - 2 alças & 452 & 72,5 & 162 & 68,9 & 290 & 74,4 & \\
\hline Costas - 1 alça & 76 & 12,2 & 36 & 15,3 & 40 & 10,3 & \\
\hline Braços e mãos & 95 & 15,3 & 37 & 15,7 & 58 & 15,0 & \\
\hline Peso do material escolar & & & & & & & 0,222 \\
\hline Adequado $(\leq 10 \%)$ & 436 & 70,3 & 172 & 39,4 & 264 & 60,6 & \\
\hline Inadequado (>10\%) & 184 & 29,7 & 63 & 34,2 & 121 & 65,8 & \\
\hline Práticas sedentárias & & & & & & & 0,349 \\
\hline Sim & 143 & 22,9 & 49 & 34,3 & 94 & 65,7 & \\
\hline Não & 482 & 77,1 & 186 & 38,6 & 296 & 61,4 & \\
\hline Atividade física na escola & & & & & & 0,749 & \\
\hline Sim & 485 & 86,8 & 193 & 39,8 & 292 & 60,2 & \\
\hline Não & 74 & 13,2 & 28 & 37,8 & 46 & 62,2 & \\
\hline $\begin{array}{l}\text { Atividade física fora do } \\
\text { ambiente escolar }\end{array}$ & & & & & & 0,446 & \\
\hline $\operatorname{sim}$ & 379 & 60,6 & 138 & 36,4 & 241 & 63,6 & \\
\hline Não & 246 & 39,4 & 97 & 39,4 & 149 & 60,6 & \\
\hline Prática de esporte & & & & & & 60,5 & 0,263 \\
\hline Sim & 334 & 53,5 & 132 & 39,5 & 202 & & \\
\hline Não & 290 & 46,5 & 102 & 35,2 & 188 & 64,8 & \\
\hline Esporte de competição & & & & & & & $<0,001$ \\
\hline Sim & 106 & 17,3 & 58 & 54,7 & 48 & 45,3 & \\
\hline Não & 508 & 82,7 & 172 & 33,9 & 336 & 66,1 & \\
\hline Tabagismo & & & & & & & 0,068 \\
\hline Sim & 17 & 2,7 & 10 & 58,5 & 7 & 41,2 & \\
\hline Não & 607 & 97,3 & 225 & 37,1 & 382 & 62,9 & \\
\hline
\end{tabular}


$\mathrm{Na}$ Tabela 2 estão apresentados os resultados da análise bruta e ajustada da associação da DME com os fatores investigados que apresentaram valor de $\mathrm{p}$ menor que 0,20 na análise multivariável. As variáveis idade, sexo, histórico familiar de distúrbios da coluna, esportes de competição e tabagismo apresentaram associação significativa com o desfecho na análise bruta. Contudo, na análise ajustada a variável tabagismo perdeu sua associação com DME.

Os escolares com idade entre 11 e 18 anos, apresentaram um risco 70\% maior de apresentar DME quando comparados aos escolares de seis e dez anos.
Quanto ao sexo, as meninas apresentaram um risco cerca de $70 \%$ maior do que nos meninos.

A história familiar de distúrbio de coluna mostrou-se associado ao desfecho, sendo o risco maior $(p \leq 0,001)$ entre as crianças cujos pais apresentavam esses sintomas.

O fato do escolar não realizar esporte de competição mostrou-se como um fator de proteção para o desfecho $(p \leq 0,001)$.

A variável tabagismo não apresentou associação significativa com o desfecho na análise ajustada $(p=0,192)$.

\section{Tabela 2}

Análise bruta e ajustada da associação da dor musculoesquelética (DME) com variáveis selecionadas em escolares ( $\mathrm{N}=625)$ do $1^{\circ}$ ao $9^{\circ}$ ano do município de Rio Grande, RS, 2011.

\begin{tabular}{|c|c|c|c|c|c|c|c|c|c|}
\hline \multirow[t]{3}{*}{ Níveis hierárquicos } & \multirow[t]{3}{*}{ Variável } & \multicolumn{4}{|c|}{ DME } & \multirow{3}{*}{$\begin{array}{l}\text { Análise bruta } \\
\text { RP (IC95\%) }\end{array}$} & \multirow[t]{3}{*}{$p$} & \multirow{3}{*}{$\begin{array}{l}\text { Análise ajustada } \\
\text { RP (IC95\%) }\end{array}$} & \multirow[t]{3}{*}{$p$} \\
\hline & & \multicolumn{2}{|c|}{ Sim } & \multicolumn{2}{|c|}{ Não } & & & & \\
\hline & & $\mathrm{n}$ & $\%$ & $\mathrm{n}$ & $\%$ & & & & \\
\hline \multirow[t]{6}{*}{$1^{\circ}$ Nível } & Idade (anos) & & & & & & 0,001 & & 0,001 \\
\hline & 6-10 & 76 & 32,3 & 202 & 51,8 & 1,00 & & 1,00 & \\
\hline & $11-18$ & 159 & 67,7 & 188 & 48,2 & $1,68(1,31-2,09)$ & & $1,70(1,36-2,11)$ & \\
\hline & Sexo & & & & & & 0,001 & & 0,001 \\
\hline & Masculino & 86 & 36,6 & 198 & 50,8 & 1,00 & & 1,00 & \\
\hline & Feminino & 149 & 63,4 & 192 & 49,2 & $1,44(1,16-1,79)$ & & $1,47(1,19-1,81)$ & \\
\hline \multirow[t]{3}{*}{$2^{\circ}$ Nível } & $\begin{array}{l}\text { Histótico familiar distúr- } \\
\text { bios da coluna }\end{array}$ & & & & & & 0,001 & & 0,020 \\
\hline & Não & 113 & 48,7 & 243 & 62,5 & 1,00 & & 1,00 & \\
\hline & Sim & 119 & 51,3 & 146 & 37,5 & $1,41(1,15-1,73)$ & & $0,78(0,64-0,96)$ & \\
\hline \multirow[t]{6}{*}{$3^{\circ}$ Nível } & Esportes de competição & & & & & & 0,001 & & 0,001 \\
\hline & Sim & 58 & 25,2 & 48 & 12,5 & 1,00 & & 1,00 & \\
\hline & Não & 172 & 74,8 & 336 & 87,5 & $0,62(0,50-0,76)$ & & $0,65(0,51-0,81)$ & \\
\hline & Tabagismo & & & & & & 0,038 & & 0,192 \\
\hline & Não & 225 & 95,7 & 382 & 98,2 & 1,00 & & 1,00 & \\
\hline & Sim & 10 & 4,3 & 7 & 1,8 & $1,58(1,01-2,39)$ & & $1,36(0,85-2,17)$ & \\
\hline
\end{tabular}




\section{Discussão}

Os resultados do estudo mostraram que a prevalência de dor musculoesquelética encontra-se dentro dos percentuais descritos na literatura, que variam de $12,2 \%$ a $40 \% .5,20$ Esta elevada prevalência de DME pode vir a desencadear diferentes problemas osteomusculares, tais como alterações posturais, dificultar o processo de aprendizagem, bem como a realização das atividades da vida diária. ${ }^{13}$

No presente estudo, a análise multivariada mostrou que idade, sexo, histórico familiar de dor nas costas e prática de esportes de competição estiveram associados à ocorrência de DME entre os escolares.

A prevalência de DME foi quase duas vezes maior nos escolares com idade entre 11 e 18 anos quando comparada aos com idade entre seis e dez anos. Esses resultados corroboram com estudos prévios que mostraram a elevação da frequência de dor musculoesquelética com o aumento a idade. 5,21 Acredita-se que esta associação possa ser justificada pelo aumento do tempo de exposição a mochilas com excesso de peso que desencadeiam acúmulo de sobrecarga sobre as estruturas musculoesqueléticas. 13 Smith e Leggat,22 revisando a literatura, propõem que a maior prevalência de DME em escolares com maior idade pode estar relacionada à mudanças de hábitos ocorridos com o aumento da idade, como o uso mais frequente de computadores e videogames.

Quanto à maior ocorrência de DME nas meninas do que nos meninos observada neste estudo, essa associação foi observada por outros autores e tem sido atribuída à fatores como puberdade, crescimento rápido, dores na região da posterior do tronco e alterações na coluna vertebral.13 El-Metwally et al. 23 examinando uma coorte de 1756 escolares na Finlândia encontrou uma chance $40 \%$ maior de aparecimento de dor na coluna entre as meninas. De Vitta et al. ${ }^{6}$ relatam associação semelhante à encontrada neste estudo, atribuindo os resultados à diferença de força muscular entre os gêneros, sendo menor no sexo feminino, característica que aumenta o risco de sobrecarga musculoesquelética.

Este estudo não encontrou associação significativa entre DME e excesso de peso. Um estudo em escolares brasileiros com idade entre seis e 12 anos, que relaciona o índice de massa corporal à dor musculoesquelética encontrou resultado semelhante. 24 Contudo, Deere et al., $25 \mathrm{em}$ seu estudo de base populacional com 3376 adolescentes, encontraram associação entre excesso de peso e presença de dor musculoesquelética. Estudo realizado na
Alemanha sobre a influência do excesso de peso no sistema musculoesquelético relata que o sobrepeso e/ou obesidade conduz a sobrecarga nas articulações, e consequentemente nas placas de crescimento, ocasionando alterações no desenvolvimento do esqueleto axial, e em toda a estrutura musculoesquelética. ${ }^{11}$

Acreditamos que a ausência de associação entre excesso de peso e o desfecho pode ter sido devido à elevada homogeneidade da população estudada quanto as características do estado nutricional, a maioria classificada como eutrófico/baixo peso, a qual não permitiu análises comparativas.

Com relação à associação encontrada entre DME e a presença de histórico familiar de dores na coluna, estes achados corroboram com os resultados relatados na literatura. 26,27 Masiero et al.27 em pesquisa realizada com 7542 adolescentes italianos encontraram associação entre presença de dor lombar entre escolares e histórico familiar de dor lombar. Contudo, cabe ressaltar que a literatura encontrada está baseada em estudos entre adolescentes e com desfecho de dor lombar, diferentemente do presente estudo que inclui escolares entre seis e 18 anos.

As variáveis relacionadas às características do suporte para transporte do material escolar não mostraram associação significativa com o desfecho na análise multivariada. Contudo, o peso da mochila escolar, seu modelo e o modo de transporte têm sido identificados por outros autores como fatores de risco importantes para o surgimento do DME nos escolares.28,29 Esses autores ressaltam que o peso excessivo da mochila e a maneira de transporte do material escolar ocasiona uma inclinação do corpo do escolar para o lado ou para frente, contribuindo para o surgimento de curvaturas laterais, inclusive na região lombar da coluna vertebral, que por sua vez desencadeiam dores na coluna destes indivíduos. 28,29

Acredita-se que o fato das escolas pertencerem exclusivamente à rede municipal e com predomínio de estudantes com nível socioeconômico baixo, possivelmente restrinja a aquisição e uso de material escolar, justificando a ausência de associação da DME com o modo de transporte do material escolar. Verificou-se que a prática de esportes de competição apresentou associação positiva com o desfecho estudado, uma vez que pode gerar sobrecarga no sistema musculoesquelético ainda imaturo dos escolares. Também é importante salientar que o interesse pela prática de esportes de competição é maior entre os dez e 13 anos de idade, quando ocorre o estirão de crescimento, e nesta fase da vida isto pode resultar 
em desalinhamentos e adaptações em toda a estrutura muscular e óssea do indivíduo, 30 e consequentemente, DME. No entanto, ressalta-se que este estudo não levantou informações sobre o tipo de esporte praticado, a frequência e intensidade do treinamento e, portanto, tais resultados apresentam fragilidade. De qualquer forma, ressalta-se a importância da atenção especial às crianças que praticam esportes de competição, de forma a prevenir distúrbios que possam comprometer a saúde musculoesquelética, inclusive na vida adulta.

Neste estudo não foi encontrada associação entre excesso de peso da mochila e DME. Entretanto,

\section{Referências}

1. Grimes P, Legg S. Musculoskeletal Disorders (MSD) in school students as a risk factor for adult MSD: A review of the multiple factors affecting posture, comfort and health in classroom environments. J Human-environmental Syst. 2007; 7 (1): 1-9.

2. Cardon G, Balagué F. Low back pain prevention's effects in schoolchildren. What is the evidence? Eur Spine J. 2004; 13 (1): 663-79.

3. De Inocencio J. Epidemiology of musculoskeletal pain in primary care. Arch Dis Child. 2004; 89: 431-4.

4. Olsen TL, Andetson RL, Deanwate SR, Kiska AM, Cauley JA, Aaron DJ, Laporte RE. The epidemiology of low back pain an adolescent population. Am J Public Health. 1992; 82 (4): 605-8.

5. Zapata AL, Moraes AJP, Leone C, Doria-Filho U, Silva CAA. Pain and musculoskeletal pain syndromes related to computer and video game use in adolescents. Eur J Pediatr. 2006; 165: 408-14.

6. De Vitta A, Martinez MG, Piza NT, Simeão SFA, Ferreira NP. Prevalence of lower back pain and associated factors in students. Cad Saúde Pública. 2011; 27: 1520-8.

7. Mcbeth J, Jones K. Epidemiology of chronic musculoskeletal pain. Best Pract Res Clin Rheumatol. 2007; 21 (3): 403-25.

8. Timpka S, Petersson IF, Zhou C, Englund M. Muscle strength in adolescent men and future musculoskeletal pain: a cohort study with 17 years of follow-up. BMJ Open. 2013; 28 (3): 1-16.

9. Rhee H. Racial/ethnic differences in adolescents physical symptoms. J Pediatr Nurs. 2005; 20 (3): 153-62.

10.Stovitz SD, Pardee PE, Vazquez G, Duval S, Schwimmer JB. Musculoskeletal pain in obese children and adolescents. Acta Pædiatra. 2008; 97 (4): 489-93.

11. Schönau E. Kindliche adipositas - folgen für den Bewegungsapparat und Therapieansätze. Bundesgesundheitsb1. 2013; 56: 528-31.

12.Palmer KT, Syddall H, Cooper C, Coggon D. Smoking and musculoskeletal disorders: findings from a British national survey. Ann Rheum Dis. 2003; 62: 33-6.

13. Noll M, Candotti CT, Tiggeman CL, Schoenell MCW, ressalta-se que esse dado possui limitações, desde que o peso da mochila foi aferido em um único momento o que gera distorções, considerando que a quantidade de material escolar varia de acordo com a grade de disciplinas do estudante.

Finalmente, considera-se a implantação de programas de promoção da saúde nas escolas uma medida importante, as quais estimulem a adoção de práticas saudáveis pelos estudantes e prevenindo os hábitos nocivos à saúde como o tabagismo e o uso da televisão, computador ou jogos de videogame por longos períodos.
Vieira A. Prevalência de dor nas costas e fatores associados em escolares do ensino fundamental do município de Teutônia, Rio Grande do Sul. Rev Bras Saúde Matern Infant. 2012; 12 (4): 395-402.

14.Diepenmaat AC, Van der Wal MF, de Vet HC, Hirasing RA. Neck/shoulder, low back, and arm pain in relation to computer use, physical activity, stress, and depression among dutch adolescents. Pediatrics. 2006; 117 (2): 412-6.

15.Coleman J, Straker L, Ciccarelli M. Why do children think they get discomfort related to daily activities? Work. 2009; 32 (1): $267-74$.

16. Gonçalves FD, Catrib AMF, Vieira NFC, Vieira LFES. A promoção da saúde na educação infantil. Interface (Botucatu). 2008; 12 (24): 181-92.

17. IBGE (Instituto Brasileiro de Geografia e Estatística). [acesso em jun 2014]. Disponível em: http/www.ibge.gov.br/home

18.Pinheiro FA, Trócolli BT, Carvalho CV. Validação do questionário nórdico de sintomas osteomusculares como medida de morbidade. Rev Saúde Pública. 2002; 36 (3): 307-12.

19.Onis M, Onyango AW, Borghi E, Siyam A, Nishida C, Siekmann J. Development of a WHO growth reference for school-aged children and adolescents. Bulletin of the World Health Organization. 2007; 85 (9): 660-7.

20.Kistner F, Fiebert I, Roach K, Moore J. Postural Compensations and subjective complaints due to backpack loads and wear time in schoolchildren. Pediatr Phys Ther. 2013; 25 (1): 15-24.

21.King S, Chambers CT, Huguet A, MacNevin RC, McGrath PJ, Parker L, MacDonald AJ. The epidemiology of chronic pain in children and adolescents revisited: a systematic review. Pain. 2011; 152: 2729-38.

22.Smith DR, Leggat PA. Back pain in the young: a review of studies conduct among school childrens and university students. Current Pediatr Reviews. 2007; 3 (1): 69-77.

23.El-Metwally A, Salminen JJ, Auvinen A, Macfarlane G, Mikkelsson M. Risk factors for development of nonspecific musculoskeletal pain in preteens and early adolescents: a prospective 1-year follow-up study. BMC Musculoskelet Disord. 2007; 8: 1-8. 
24.Pereira DSL, Castro SS, Bertoncello D, Damião R, Walsh IAP. Relação da dor musculoesquelética com variáveis físicas. Braz J Phys Ther. 2013; 17 (4): 392-400.

25.Deere KC, Clinch J, Holliday K, McBeth J, Crawley EM, Sayers A, Palmer S, Doerner R, Clark EM, Tobias JH Obesity is a risk factor for musculoskeletal pain in adolescents: findings from a population-based cohort. Pain. 2012; 153 (9): 1932-8

26.Power C, Frank J, Hertzman C, Schierhout G, Li L. Predictors of low back pain onset into a prospective British study. Am J Public Health. 2001; 91 (10): 1671-8.

27. Masiero S, Carraro E, Celia A, Sarto D, Ermani M. Prevalence of nonspecific low back pain in schoolchildren aged between 13 and 15 years. Acta Paediatr. 2008; 97 (2): $212-6$
28.Aparicio EQ, Nogueras AMM, Sendín NL, Alonso ABR, Pedraz LS, Arenillas JIC. Influencia del tipo de jornada escolar en el peso de lãs mochilas escolares. Fisioterapia. 2005; 27 (1): 6-15.

29.Shamsoddini AR, Hollisaz MT, Hafezi R. Backpack weight and musculoskeletal symptoms in secondary school students, Tehran, Iran. Iran J Publ Health. 2010; 39 (4): 120-5.

30.Korovessis P, Koureas G, Zacharatos S, Papazisi R. Backpack, back pain, sagital spine curves and trunk alignment in adolescents: a logistic and multinomial logistic analysis. Spine. 2005; 30 (2): 247-55.

Recebido em 13 de setembro de 2013

Versão final apresentada em 20 de maio de 2014

Aprovado em 18 de junho de 2014 